\title{
Effects of power truncation on the insertion loss and crosstalk of arrayed-waveguide grating devices
}

\author{
A. A. Bernussi, ${ }^{\text {a) }}$ L. Grave de Peralta, S. Frisbie, and H. Temkin \\ Department of Electrical and Computer Engineering, Texas Tech University, Lubbock, Texas 79409
}

(Received 17 December 2003; accepted 30 June 2003)

\begin{abstract}
A measurement of the insertion loss and crosstalk in folded, $100 \mathrm{GHz}$, arrayed waveguide multiplexers as a function of the number of grating waveguides is described. The number of grating waveguides can be varied in a single device to evaluate power truncation effects with high accuracy. We show that the central peak insertion loss decreases exponentially with the number of grating waveguides. The crosstalk decreases with increased number of waveguides and the measured dependence shows valleys and peaks associated with the passband spectrum of individual channels. These observations are in good agreement with simulation results. With the arrayed waveguide design used in this work the crosstalk performance becomes phase error limited for the number of waveguides greater than 250. (C) 2003 American Institute of Physics. [DOI: 10.1063/1.1605802]
\end{abstract}

Arrayed waveguide gratings (AWGs) devices are key components in wavelength division multiplexed optical networks. ${ }^{1}$ The performance of AWGs, measured by crosstalk and insertion losses, depends on the device design parameters, the choice of waveguide materials, and on the device fabrication. ${ }^{2}$ The limited number of grating waveguides, or power truncation, affects the crosstalk limit of AWGs, and to some extent, their insertion loss. In addition, imperfections of the device fabrication, or phase errors, contribute to further deterioration of the crosstalk. ${ }^{3,4}$ Consequently, in order to obtain high performance AWGs it is essential to understand the effect of power truncation and phase errors.

In this work we present an experimental investigation of crosstalk and loss dependence of folded AWGs on the number of grating waveguides. Our method allows us to vary the number of waveguides in the grating in a single device eliminating the need for the design and fabrication of multiple devices. Our results show that losses decrease exponentially and tend to saturate at high numbers of grating waveguides. We also observe a decrease in crosstalk and the measured decrease exhibits well-defined valleys and peaks. Large numbers of waveguide gratings result in lower values of crosstalk. For more than 250 waveguides in a grating the crosstalk becomes limited by random phase errors. The experimental results are in good agreement with the calculated dependence of the insertion loss and crosstalk on the number of waveguides in the grating.

The AWGs used in this work are folded by the introduction of a reflecting surface in the grating region. The device is designed for 40-channel operation with the channel-tochannel spacing of $100 \mathrm{GHz}$ and it is fabricated using $\mathrm{SiO}_{2}$ layers deposited on a silicon substrate. The waveguide relative refractive index $(n)$ difference $\left(n_{\text {core }}=1.456\right.$ and $n_{\text {cladding }}=1.446$, @ $\left.1.55 \mu \mathrm{m}\right)$ between the core and the cladding materials is $0.68 \%$. All the devices used in this study were fabricated with the nominal photolithographic mask resolution and placement accuracy of $0.1 \mu \mathrm{m}$.

${ }^{a)}$ Electronic mail: ayrber@msn.com
The folded design enables one to investigate the effects of the number of grating waveguides $\left(N_{\mathrm{wg}}\right)$ on the spectral output characteristics of a single AWG. This is done by controlling the number of waveguides at the reflecting surface plane terminating the grating. Only the waveguides terminated with the reflecting surface contribute to the capture of the input power. This corresponds to power truncation of the arrayed waveguide grating. ${ }^{2,3}$ In order to experimentally investigate the effects of power truncation on the crosstalk and losses in a single AWG, the following procedure was adopted. The output characteristics of a folded AWG with a $\mathrm{Cr}-\mathrm{Au}$ reflecting film deposited at the reflecting surface were evaluated first. The deposited mirror was then removed by diamond polishing and a triangular-shaped gold mirror was used instead as an external reflector. In this configuration, the number of grating waveguides contributing to the capture of the input power can be controlled by vertical translation of the external mirror. The external mirror and the AWG were placed on three-axis angular rotation and linear translation stages, respectively. A top-view charge coupled device camera was used to monitor the position and to control the gap distance between the external mirror and the device. Careful adjustments were performed in order to minimize angular misalignments and to place the mirror and the AWG parallel to each other. Index matching fluid was applied between the external mirror and the AWG in order to minimize losses at the reflecting plane and to suppress reflections from the waveguide-air interface. A comparison between the device performances with external mirror covering all the grating waveguides and the original data revealed no appreciable difference.

Figure 1 shows representative output spectra from a single channel of a folded AWG for different values of $N_{\mathrm{wg}}$. With increasing $N_{\text {wg }}$ the principal maximum (central peak) becomes sharper and the peak losses decrease, tending to saturate for larger values of waveguides. The subsidiary maxima (sidelobes) become more numerous, less intense and shift toward the central peak. Similar results were obtained for other output channels. With increasing $N_{\text {wg }}$ a larger fraction of the optical input power is collected. This results not 


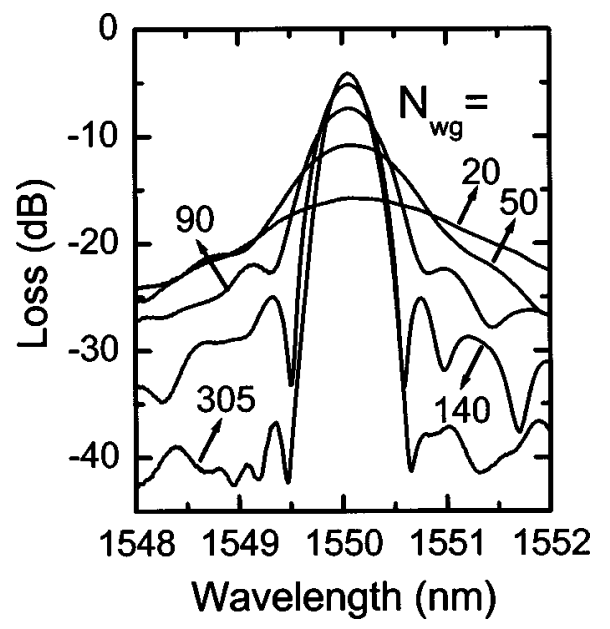

FIG. 1. Output spectra of a single channel from a folded AWG as a function of number of grating waveguides.

only in a pronounced decrease of the main peak loss but also modifies the spectral characteristics of the sidelobes, and that in turn affects the crosstalk performance of the device.

Figure 2 shows the detailed loss dependence of the channel's central peak on the $N_{\mathrm{wg}}$. While insertion losses decrease exponentially with $N_{\text {wg }}$ we also observe a rapid decrease for small $N_{\mathrm{wg}}$ of less than 100 . This reflects the fast increase of the fraction of the input power that is collected by the grating waveguides. Larger values of $N_{\mathrm{wg}}(>100)$ resulted in slower changes. The losses continue to decrease up to the maximum number of waveguides used in our experiment, $N_{\mathrm{wg}}=305$, but the rate becomes quite low. The loss changes by less than $0.5 \mathrm{~dB}$ as $N_{\mathrm{wg}}$ increases from 170 to 305.

The AWG crosstalk performance is known to be influenced by the number of grating waveguides. ${ }^{2,3}$ Figure 3 shows the measured crosstalk between adjacent channels of the AWG device analyzed in Fig. 1. The important features of the data of Fig. 3 are the strong dependence of the crosstalk on $N_{\mathrm{wg}}$ and the presence of well-resolved and reproducible peaks and valleys. For small values of $N_{\mathrm{wg}}$, below 70, we observe high crosstalk resulting from the intersection of the central peak, broadened by the small number

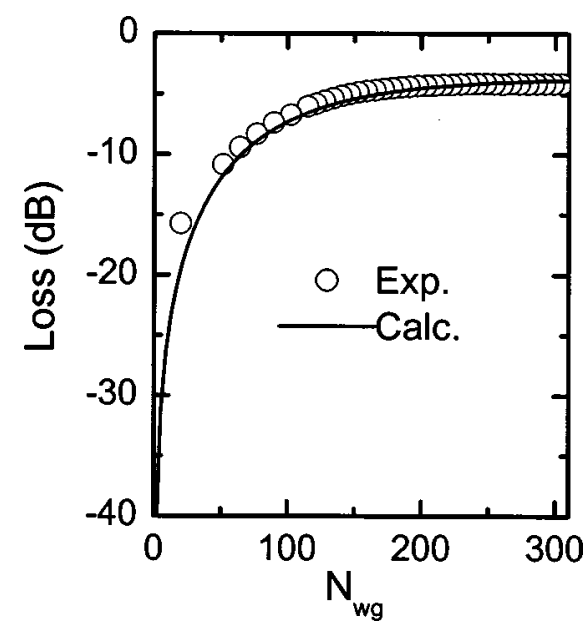

FIG. 2. Calculated (solid line) and measured $(\bigcirc)$ central peak insertion losses dependence on the number of grating waveguides for the same device shown in Fig. 1.

Using these AWG parameters and the model of Muñoz
shown in Fig. 1 .
Downloaded 13 Aug 2005 to 129.118.19.59. Redistribution subject to AIP license or copyright, see http://apl.aip.org/apl/copyright.jsp

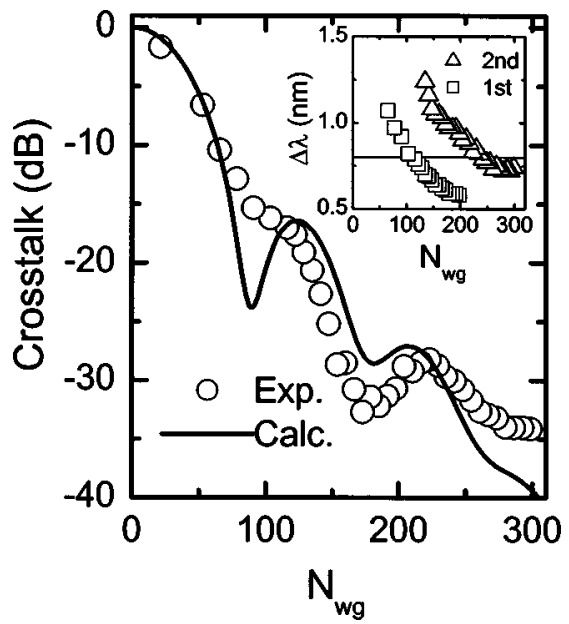

FIG. 3. Calculated (solid line) and measured $(\bigcirc)$ crosstalk as a function of the number of grating waveguides for the same device shown in Fig. 1. The inset shows the wavelength difference between the central peak and the peaks of the first two nearest sidelobes. The horizontal line in the inset corresponds to the wavelength separation to the adjacent device channel.

of waveguides (see Fig. 1), with the adjacent channel. With increased $N_{\mathrm{wg}}$ an inflection in the crosstalk data is observed around $N_{\text {wg }} \sim 110$ and another for $N_{\text {wg }} \sim 175$. This is followed by a crosstalk peak for $N_{\mathrm{wg}} \sim 225$. For $N_{\mathrm{wg}}>250$ the measured crosstalk saturates at $\sim-35 \mathrm{~dB}$.

The local maxima and minima in the measured crosstalk response with increasing $N_{\mathrm{wg}}$ are associated with wavelength and intensity of the sidelobe peaks. As shown in Fig. 1 the nearest sidelobe moves toward the central peak with increasing $N_{\mathrm{wg}}$. When the peak position of the valley (peak) of the sidelobe coincides with the center of the adjacent channel, the crosstalk exhibits a valley (peak). In order to illustrate this effect the wavelength difference $(\Delta \lambda)$ between the experimental central peak position and the peak positions of the two nearest sidelobes are plotted in the inset of Fig. 3 as a function of $N_{\mathrm{wg}}$. When the sidelobe peak position crosses the corresponding wavelength separation between adjacent channels (solid line in the inset) the crosstalk exhibits a peak. This effect is more pronounced for the crosstalk peak at $N_{\text {wg }} \sim 225$, when compared to the one observed at $N_{\text {wg }} \sim 110$, due to the presence of well-resolved sidelobes in the range $110<N_{\text {wg }}<250$ (see Fig. 1).

In order to analyze the spectral output characteristics of the AWG as a function of the number of grating waveguides, we calculated the device response using the AWG field model of Muñoz et al., 5 in the Gaussian approximation, and the following device parameters. A constant length difference value of $\Delta L=12.3 \mu \mathrm{m}$ between grating waveguides was used in our AWG design. This corresponds to a diffraction order of $m=23$. This small $\Delta L$ value enables the use a large number of grating waveguides with separation between waveguides at the slab arc of $25.6 \mu \mathrm{m}$. The number of grating waveguides in our design is 305 . In order to achieve loss nonuniformity of $0.5 \mathrm{~dB}$ a free frequency spectral range of $8.4 \mathrm{THz}$ was chosen. Tapered waveguides of $22.5 \mu \mathrm{m}$ width were used in the grating region. A slab radius of $5 \mathrm{~cm}$ was used together with tapered waveguides of $9.6 \mu \mathrm{m}$ width at the input/output coupler region in order to achieve a $-3 \mathrm{~dB}$ channel bandwidth of at least $0.4 \mathrm{~nm}$. 
et $a{ }^{5}{ }^{5}$ we can compare simulated and measured central peak losses as a function of the number of grating waveguides, as shown in Fig. 2. A good agreement between theory and experiment is clear. Qualitative agreement between the measured and calculated crosstalk is also obtained, as shown in Fig. 3. The overall trend observed in experimental and calculated curves is the decrease of the crosstalk with increasing $N_{\text {wg }}$, in agreement with the general concept of reduced crosstalk for larger gratings. ${ }^{2}$ For $N_{\mathrm{wg}}<50$ the calculated and measured channel response is broad and the central peak extends into the adjacent channel. The sidelobes are spectrally well separated and do not contribute to the channel-tochannel crosstalk. For $N_{\mathrm{wg}}>50$ the nearest sidelobe minimum first shifts into the adjacent channel, resulting in a drop in the calculated crosstalk, and then shifts out, with a corresponding increase in the crosstalk. The experimental resolution of the grating is low for low $N_{\mathrm{wg}}$ resulting in relatively poor match with the calculated crosstalk. Resolution improves with increasing $N_{\mathrm{wg}}$ and a better agreement for $N_{\text {wg }} \sim 175$ is obtained.

The calculated crosstalk continues to decrease with increasing $N_{\mathrm{wg}}$. In contrast, the measured crosstalk saturates at about $-35 \mathrm{~dB}$. This is attributed to optical phase errors in the arrayed waveguides. ${ }^{3,4}$ Phase errors arise from material and device processing inhomogeneities (thickness and refractive index, waveguide width, etc.), and deviations from the ideal path-length difference caused by resolution limitations in the photolithographic process. $^{3}$ Random phase errors affect mainly the sidelobe valleys of the output spectra and consequently the crosstalk. Unlike the sidelobes, the central peak is less influenced by the phase errors. This is consistent with the results of Fig. 2 where a good agreement between simulated and measured peak losses as a function of $N_{\text {wg }}$ was obtained. It is also possible that phase errors contribute to the difference between the measured and calculated crosstalk for $N_{\text {wg }} \sim 100$. This is because the relative importance of phase errors is larger for smaller $N_{\mathrm{wg}} \sim 100$.

In summary, we investigated power truncation effects on the crosstalk and loss performances of folded AWGs. The folded design allowed direct investigation, with the use of an external reflector, of the effect of the number of grating waveguides in a single device. This allowed us to keep the overall device design constant and vary only the number of waveguides. Channel output losses were found to decrease exponentially with the number of grating waveguides and a good agreement between the model and data was obtained. The crosstalk performance as a function of the number of waveguides revealed well-defined peaks and valleys caused by sidelobe shifts, demonstrating the accuracy of the measurement. The crosstalk and insertion losses were modeled with the AWG field model in the Gaussian approximation. For the highest number of waveguides the crosstalk become limited by random phase errors of the fabrication process.

This work was supported by the State of Texas under the Technology Development and Transfer program, Texas Instruments, Inc., and the Jack F. Maddox Foundation.

${ }^{1}$ G. E. Keiser, Opt. Fiber Technol. 5, 3 (1999).

${ }^{2}$ K. Okamoto, Fundamentals of Optical Waveguides (Academic, New York, 2000), Chap. 9.

${ }^{3}$ C. D. Lee, W. Chen, Q. Wang, Y. J. Chen, W. T. Beard, D. Stone, R. F. Smith, R. Mincher, and I. R. Stewar, J. Lightwave Technol. 19, 1726 (2001).

${ }^{4}$ H. Yamada, H. Sanjoh, M. Kohtoku, K. Takada, and K. Okamoto, J. Lightwave Technol. 18, 1309 (2000).

${ }^{5}$ P. Muñoz, D. Pastor, and J. Capmany, J. Lightwave Technol. 20, 661 (2002). 